\title{
La transformación del suelo a uso industrial con criterios de sostenibilidad
}

\author{
María Luisa Soriano Sanz y \\ Francisco Maza Vázquez
}

\section{Resumen}

Las estrategias de desarrollo de los municipios deben de estar enmarcadas dentro de una perspectiva territorial equilibrada que promuevan criterios de sostenibilidad. La acción urbanística futura ha de ser objeto de una transformación del suelo, en el que el proceso va a estar condicionado por factores económicos, sociales y ambientales.

Para alcanzar la nueva estructura urbana adecuándose a los instrumentos urbanísticos de planeamiento y dotar al municipio de suelo con uso industrial, que pueda ponerse a disposición de los potenciales destinatarios en condiciones de oferta atractiva, se plantea un estudio metodológico que abarca desde la viabilidad técnica y económica, hasta la adquisición de suelo, la gestión, el diseño y la ejecución de la actuación urbanística para el desarrollo de la actividad económica.

Palabras clave: Desarrollo. Municipio. Suelo industrial. Sostenibilidad.

\section{Abstract}

Development strategies of municipalities must be framed within a perspective that promotes balanced territorial sustainability criteria. The future urban planning activities must be subject to a land transformation, in which the process will be conditioned by economic, social and environmental.

To reach the new urban structure adapting to urban planning instruments and provide the municipality with industrial land, which could be made available to potential recipients on offer attractive conditions, there is a methodological study ranging from the technical and economic, to land acquisition, management, design and implementation of urban development for the development of economic activity.

Keywords: Development. Municipality. Industrial land. Sustainability. Introducción 


\section{INTRODUCCIÓN}

El desarrollo sostenible o sustentable y el medio ambiente se sitúan en centro de atención hacia finales del siglo pasado. El debate sobre "eco desarrollo" que se formuló en Estocolmo, en el año 1972, y la "Cumbre de la Tierra", celebrada en Río de Janeiro (1992), fueron hitos relevantes en la concepción internacional de la relación entre el hombre y el medio. Desde entonces mucho se ha escrito sobre la sostenibilidad y de las herramientas que pone a nuestro alcance este término.

La Comisión Europea de Medio Ambiente (1996) apunta que el desarrollo sostenible es un concepto complejo que incorpora principios de sostenibilidad social, ambiental y económica, y además se demanda que el desarrollo sea económicamente eficiente y equitativo dentro, y entre generaciones (Carrizosa, 1993).

A veces, la expresión "desarrollo sostenible" ha estado ligada con el significado de mejorar la calidad de vida humana sin rebasar la capacidad de carga de los ecosistemas que la sustentan, así lo expone el conocido informe de "Cuidar la Tierra" publicado por la Unión Internacional para la Conservación de la Naturaleza (UICN), en 1991.

Otros autores definen la sostenibilidad como "la satisfacción de las necesidades presentes sin comprometer la capacidad de las generaciones futuras de satisfacer las suyas". (Brundtland, 1987). Sin duda esta definición y la justicia social tiene mucho que ver con el hecho de que el $26 \%$ de la población mundial consume el $85 \%$ de los recursos no renovables y el $40 \%$ de las existencias alimenticias, planteando serias consideraciones éticas y morales.

También el término de desarrollo sostenible lleva implícito un concepto dinámico que abarca el crecimiento y desarrollo económico y social dentro de los límites determinados por la naturaleza (LEADER, Magazine $\left.n^{0} 6,7 / 94\right)$. No se trata de una teoría del desarrollo ni de un modelo de crecimiento, sino de un enfoque que es imprescindible aplicar en la ordenación territorial.

Estas consideraciones previas conducen a pensar que el servicio que nos proporciona el urbanismo y la Ordenación del Territorio nos sirven para impulsar un modelo de desarrollo urbano que sea capaz de satisfacer las necesidades sociales, ambientales y económicas, garantizando la sostenibilidad de los ecosistemas naturales. En este sentido, el Informe sobre los Recursos Mundiales elaborado en el año 1996 por el Programa de Naciones Unidas para el Desarrollo (PNUD) enfoca el desarrollo sostenible como un proceso de ordenación que requiere un progreso 
simultáneo en las diversas dimensiones: económica, humana, ambiental y tecnológica.

Así mismo, el proceso de ordenamiento territorial, está necesariamente orientado al desarrollo sustentable, pues debe buscar favorecer el crecimiento económico, elevar las condiciones de vida de la población en pos de la equidad, y mantener vital y sólida la sustentabilidad de los ecosistemas y recursos naturales del territorio.

A este respecto, la también llamada sostenibilidad de un territorio, se basa en que el planeamiento urbano pueda reconocer las funciones de ciudad, de acuerdo con las clasificaciones de suelo, a saber, urbano, urbanizable, programado industrial o residencial y suelo rústico; teniendo en cuenta las extensiones superficiales previstas y ocupadas para cada una de estas tipologías edificatorias y reconociendo la positiva integración de lo urbano en lo que antes era rústico o entorno natural, o lo que es lo mismo reconociendo la evolución urbana del territorio, y como consecuencia de ello el desarrollo de la actividad económica.

Además, y como apoyo a la ordenación sustentable se requiere la redacción de un instrumento técnico-administrativo que nos dará la idea del impacto ambiental; es el denominado Proyecto de la Evaluación de Impacto Ambiental. A través de él, nos será posible tomar decisiones sobre la preservación de los recursos naturales y la defensa del medio ambiente, de una manera eficaz evitando las agresiones contra la naturaleza y proporcionando una mayor fiabilidad y confianza a las decisiones que deban adoptarse, elegir la mejor alternativa posible, que salvaguarde los intereses generales desde una perspectiva global e integrada y teniendo en cuenta los efectos derivados de la actividad proyectada (Soriano, 2010).

\section{LA SOSTENIBILIDAD EN LA ORDENACIÓN TERRITORIAL}

La Carta Europea de Ordenación del Territorio de 1983, define la Ordenación Territorial como "la expresión espacial de las políticas económicas, sociales, culturales y ecológicas de la sociedad. Es a la vez una disciplina científica, una técnica administrativa y una política concebida como un enfoque interdisciplinario y global, cuyo objetivo es un desarrollo equilibrado de las regiones y la organización física del espacio según un concepto rector".

La ordenación del uso de los espacios urbanos es una necesidad cada vez más imperante, que debe de contribuir a evitar el aumento de los conflictos propios de la competencia entre actividades excluyentes y proteger el medio ambiente de 
la contaminación ambiental y antrópica, permitiendo un desarrollo sostenible.

El urbanismo, la planificación del territorio, nos debe marcar pautas que ayuden a buscar la mejor solución y en definitiva a mejorar la calidad de vida de todos los ciudadanos que en ese territorio habiten; en todos los aspectos, sociales, económicos y ambientales, manteniendo abiertas todas las posibilidades de desarrollo de las generaciones futuras (Maza, 2003).

La planificación y el desarrollo urbano deben implicar una voluntad de sostenibilidad del territorio que se ordena, y en todo territorio urbano debe existir un principio de prevención; por lo que se hace necesario establecer políticas conscientes de las limitaciones ambientales y hacer ver que los recursos naturales pueden llegar a alcanzar situaciones irreversibles.

El objetivo final de la ordenación territorial es "conseguir una relación armónica entre el medio y los asentamientos humanos con el propósito de disminuir las desigualdades y lograr un desarrollo socialmente equilibrado, respetando el ambiente natural" (Durán, et al, 1993).

La ordenación territorial, es un proceso secuencial e iterativo que, orientado hacia objetivos a largo plazo, se desarrolla en ciclos de tres fases: análisis territorial, planificación territorial, y gestión territorial. Por extensión, las dos primeras pueden englobarse bajo el término de planificación, correspondiendo a la fase de gestión las tareas que comporta la puesta en práctica de un plan de ordenación (Gómez Orea, 1994).

Al centrar el debate entre sostenibilidad y ordenación del territorio, se cuestionan dudas sobre el control del crecimiento urbano que con fuerza arrastra el mercado inmobiliario. La visión descentralista, apoyada por las fuerzas económicas, mantiene su firmeza y apuesta para que la ordenación de la estructura de la ciudad dependa del libre mercado del suelo.

El interés se centra en conocer la naturaleza y el funcionamiento del territorio en todos los aspectos, socioeconómico, demográfico, comunicaciones..., aportando para ello medidas de aspectos sociales muy relacionados con la calidad de vida y el estado de bienestar. Se debe analizar el territorio, y su desarrollo económico, desde una perspectiva urbanística, observando las distintas calificaciones y clasificaciones de suelo que posee, hasta conseguir el equilibrio necesario para conseguir el bienestar deseado y afrontar la redefinición de aquellos ámbitos y su adaptación a nuevas realidades. 
La acción urbanizadora, necesaria para el desarrollo económico, supone, por tanto, la transformación de un recurso escaso y muy sensible: el suelo. La actividad debe ser lo menos agresiva posible con el lugar en que se produce. Pero es necesario seguir avanzando, continuar innovando. Y para ello es imprescindible realizar una reflexión de nuestra forma de trabajar, de producir e incluso de consumir, de medir cuál es el impacto ambiental de la actividad. Es un ejercicio de análisis y transparencia necesaria para que toda empresa, se comprometa con el medio ambiente de manera responsable y desde la auto exigencia. Contribuyendo a un mayor ahorro y eficiencia energética, así como a reducir su huella ecológica. Es tiempo de adoptar medidas valientes, nuestro progreso de hoy no puede ser un lastre para el bienestar de generaciones futuras.

\section{OBJETIVOS}

La crisis financiera internacional y el estallido de la burbuja inmobiliaria han puesto de relieve las limitaciones del crecimiento. La necesidad del cambio del modelo basado en el modelo productivo y de consumo, en la sostenibilidad y en el fomento de la innovación, se plantea como un desafío, en el que se ha de diseñar un plan estratégico.

En definitiva, el objetivo es el desarrollo de una estrategia territorial proactiva, basada en criterios de análisis socio-económicos, a través de estudios de rentabilidad y mejora de la eficiencia económica, así como estudios de viabilidad, localización de la demanda y la necesidad del territorio de una determinada actuación, que sustente la toma de decisiones, con el compromiso de la protección medio ambiental.

\section{DESARROLLO DE LA ACTIVIDAD ECONÓMICA. APLICACIÓN METODO- LÓGICA.}

Es de una importancia relevante el carácter económico que lleva implícito el término desarrollo sostenible. Se tiene como un reto el crecimiento económico que debe tener un país desarrollado y a veces la relación de economía-medio ambiente origina confrontación dentro del mundo de la economía (Solow, 1992). Potenciamos en esta sociedad, cada vez con más fuerza, el indicador de riqueza nacional y el crecimiento material, apareciendo como una necesidad axiomática. En esta sociedad de mercado parece importarnos más la contabilidad nacional, los flujos de renta y los indicadores macroeconómicos que el capital natural (Pearce et al, 1989; Ekins, 1986).

Habitualmente el hombre permite que las ciudades se comporten como 
organismos vivos que consumen recursos y cada vez es mayor la necesidad que debe de tener un territorio para abastecer y prestar servicios a una ciudad. El deterioro ambiental se deja ver, igualmente, por la contaminación atmosférica y acústica, por los vertidos que indudablemente afectan a nuestra salud y calidad de vida, y esto incide directamente en el desarrollo de la actividad económica.

Investigadores y estudiosos elaboran continuamente modelos matemáticos que demuestran los escasos recursos no renovables consumidos y que ahora pueden ser sustituidos por otros recursos; entre ellos cabe citar al economista rumano Dr. Nicholas Georgescu-Roegen, (1.906-1.994) precursor de la economía ecológica, que trata de "crear conciencia ecológica" frente al objetivo de maximizar beneficios.

Los científicos e investigadores deben incorporar criterios operativos que ayuden a un desarrollo sostenible. Se puede empezar aquí y ahora; en casa y en la ciudad y compete a las autoridades locales, junto a organizaciones vecinales, empresariales e industriales, los que deben adquirir la información adecuada para diseñar la mejor estrategia en cuanto al desarrollo de la actividad económica a aplicar en cada caso y un método.

\section{Aplicación metodológica}

Desde hace algunos años vemos cómo la actividad urbanística que se desarrolla en el territorio obliga a conjugar el hábitat y modelo de vida con el desarrollo sostenible. El concepto de desarrollo como mejora del "nivel de vida" ha dejado en gran parte el contenido económico de mejora de renta, conseguida ya una estabilidad general, por el de "calidad de vida" que asume otras variables. Así, en términos geográficos el concepto de desarrollo sostenible tiene diferentes interpretaciones para una aldea africana, una población latinoamericana o una nación industrializada europea. Tal vez, el término desarrollo sostenible preocupe más a un estado industrial y menos a una aldea africana.

Se propone, una primera interpretación del proceso de una actuación industrial, para conseguir un modelo integrado y sostenible. La acción metodológica podemos resumirla de manera esquemática en las siguientes fases:

4.1 Trabajos previos

4.2 Adquisición de suelo

4.3 Planeamiento

4.4 Urbanización (lleva implícita la reparcelación aprobada en el proyecto de actuación urbanística) 
Aunque estas fases no son compartimentos estancos, las influencias mutuas son constantes y no sólo de manera lineal, sino que se producen procesos iterativos, actuaciones en paralelo, etc. que requieren de una gran coordinación.

\subsection{Trabajos previos}

Como trabajo previo fundamental para cada actuación urbanística industrial, es recomendable la realización de un estudio de viabilidad técnica y económica, necesaria para saber a priori, si el proyecto va a ser viable. Es clave en esta etapa la adecuación del nivel de detalle con el plazo de realización del estudio.

Figura 1: Estudio de viabilidad económica

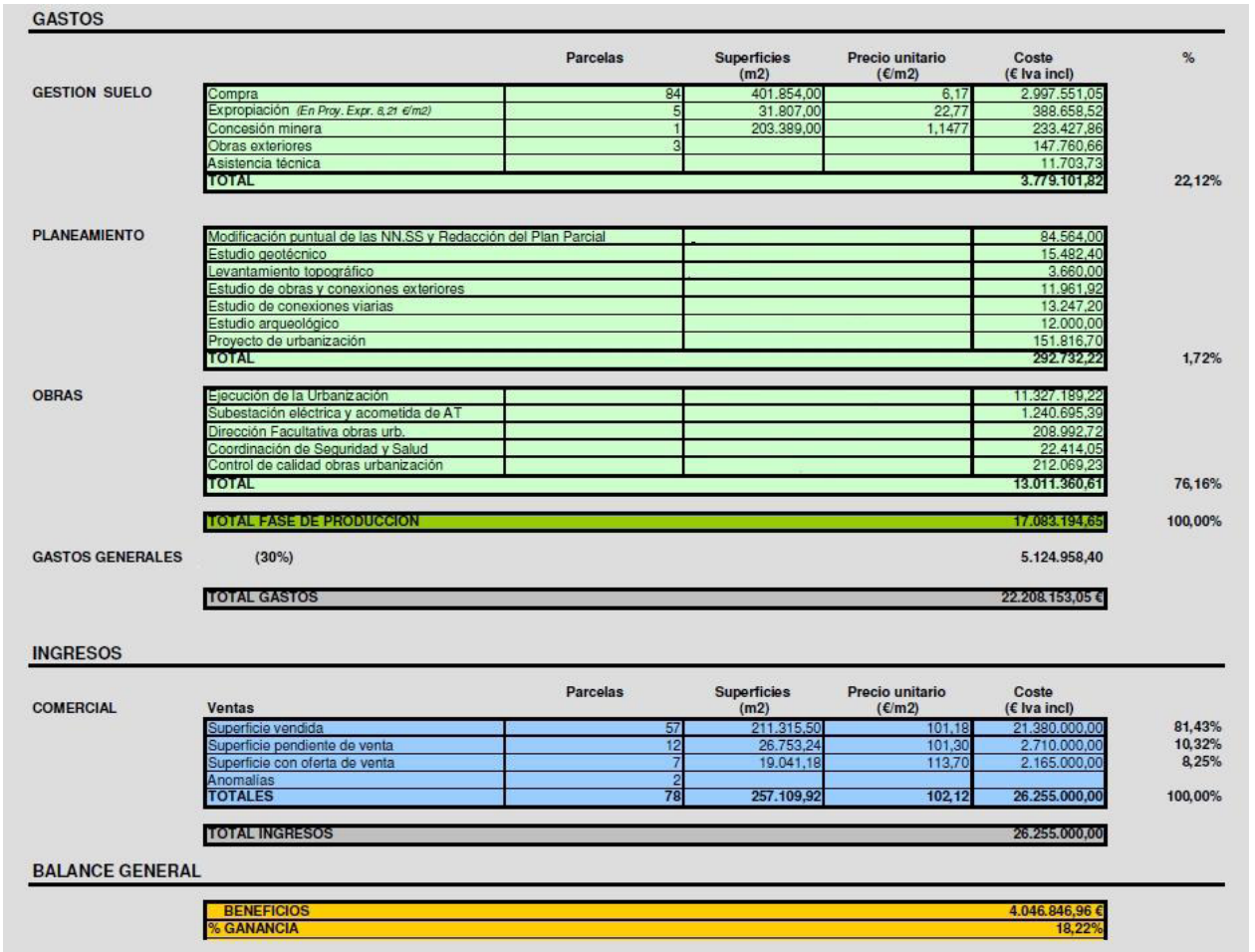


Figura 2: Fotografía aérea, para el estudio de conexiones exteriores e infraestructuras, de una futura actuación en Lentiscares, Navarrete (La Rioja)

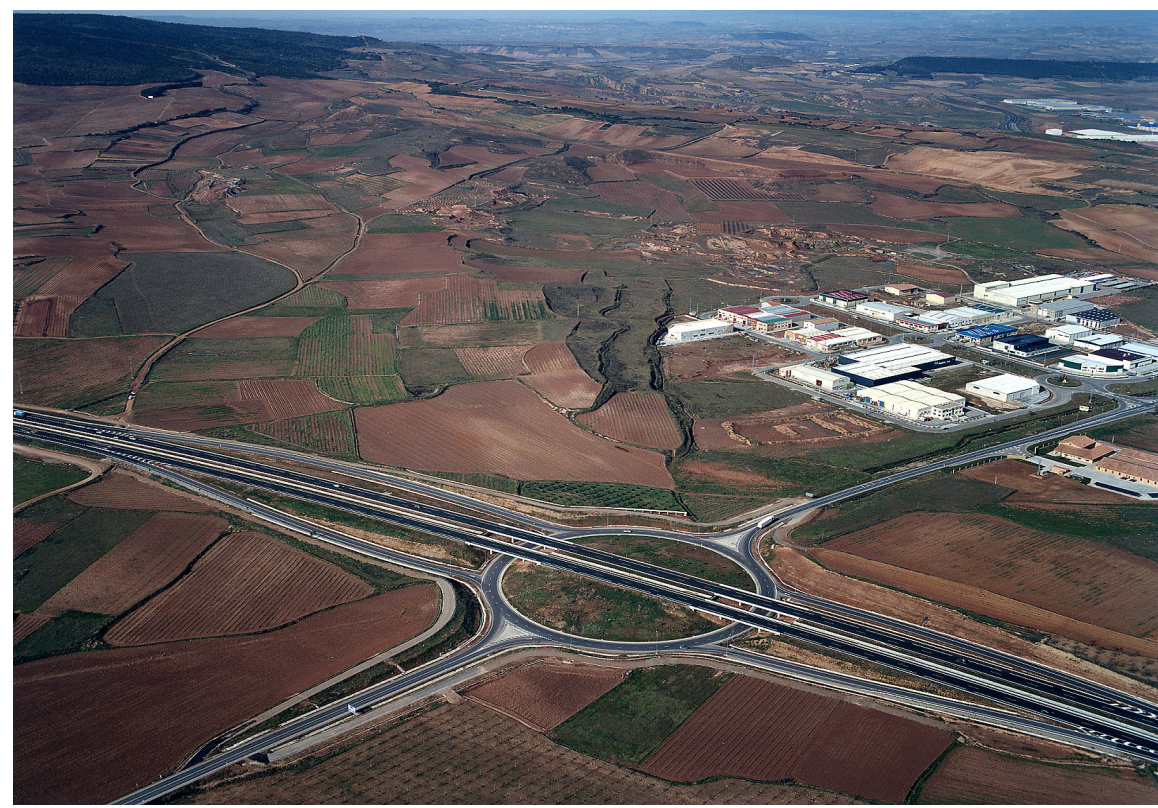

Las principales variables técnicas a estudiar son: a) posibilidad de adquisición de los terrenos, preferiblemente próximos a redes de comunicación (viaria, ferroviaria, puertos, aeropuertos...) y a núcleos de población medio-altos, y a ser posible con infraestructuras existentes en la zona (depuradora, abastecimiento, red eléctrica...), b) posible demanda de parcelas para la instalación de industrias.

En este sentido, la estructura de la sociedad ha propiciado el desarrollo de fuertes vínculos entre el individuo y el territorio. La residencia habitual está ligada a la vivienda y la actividad laboral está ligada a un centro de trabajo. La necesidad que el individuo tiene de moverse de una - la vivienda - al otro —el centro de trabajo-, exige de un sistema de comunicaciones. El individuo igualmente precisa de equipamientos sanitarios, docentes, deportivos, sociales, grandes zonas verdes, de recreo y así poder satisfacer sus necesidades, para alcanzar el ansiado desarrollo sostenible. En definitiva, el objetivo final de la ordenación territorial es una relación armónica entre el medio y los asentamientos humanos con el propósito de disminuir las desigualdades y lograr un desarrollo socialmente equilibrado, respetando el ambiente natural (Durán, et al, 1993). 
En cuanto al estudio económico, se hace aconsejable analizar la totalidad de los costes derivados de la compra o expropiación de terrenos (si es la administración quien lo fuera a ejecutar), de la producción urbanizadora (gastos de urbanización, gastos generales, financieros y beneficio empresariales), y de los ingresos potenciales de la actuación; es decir, del valor en venta de las parcelas resultantes. Para ello se deben tener en consideración los valores de repercusión correspondientes a cada uno de los usos a implantar, determinados sobre la base de un estudio de mercado actualizado, y poner de manifiesto la viabilidad positiva de la misma.

Figura 3: Parque Empresarial "Argame”. La localización del municipio de Morcín entre Mieres y Oviedo (Asturias) cuenta con potentes infraestructuras de comunicación a las que se accede a través de la carretera 630.
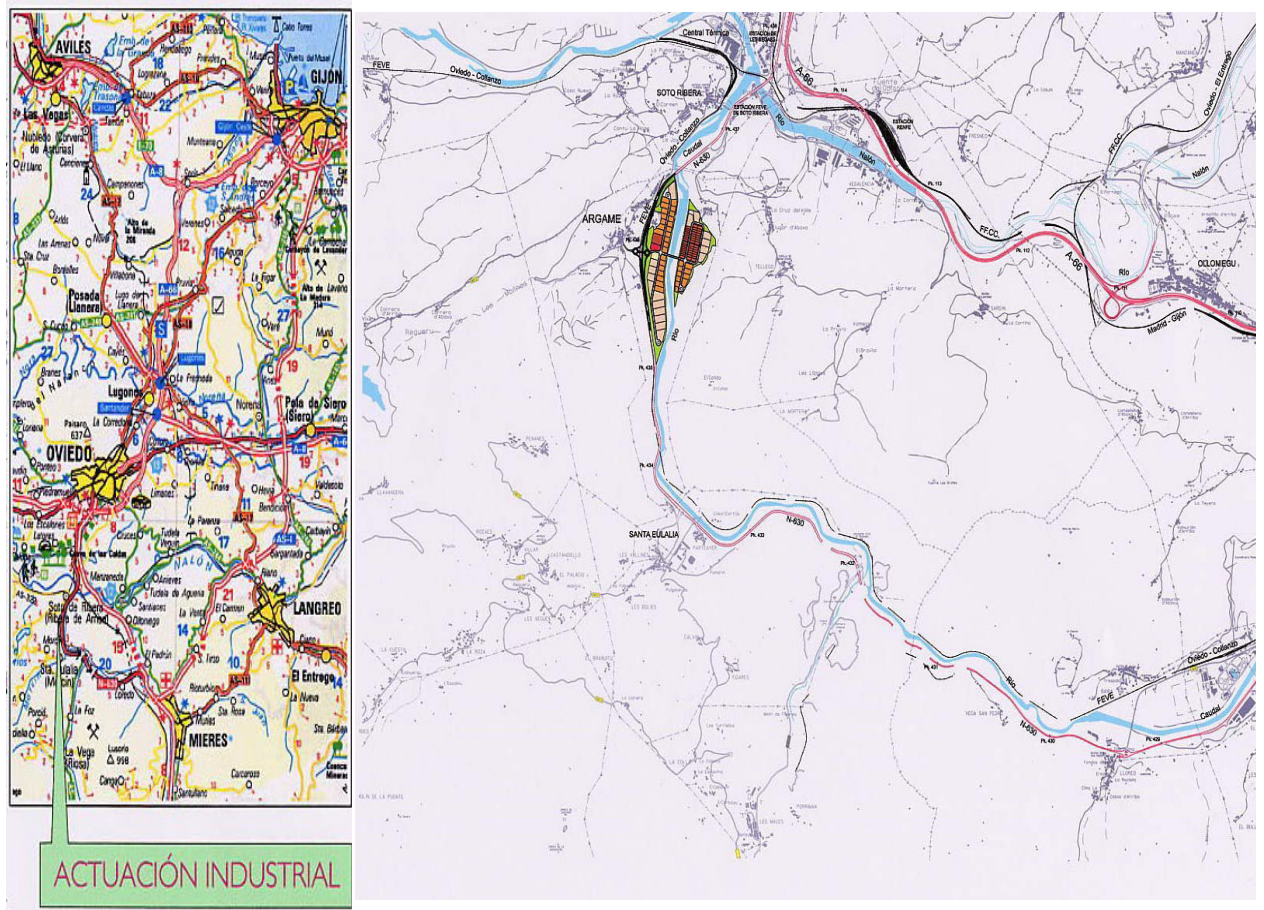

\subsection{Adquisición de suelo}

Se requiere la identificación de propietarios, bienes y derechos afectados por la actuación a partir de las bases de datos catastral y registral, que nos proporciona las delegaciones provinciales de catastro y los registros de la propiedad 
correspondientes. En la adquisición del suelo, conviene tener presente las fincas afectadas para las conexiones exteriores de infraestructuras.

Figura 4: Plano de parcelación, para la adquisición de suelo
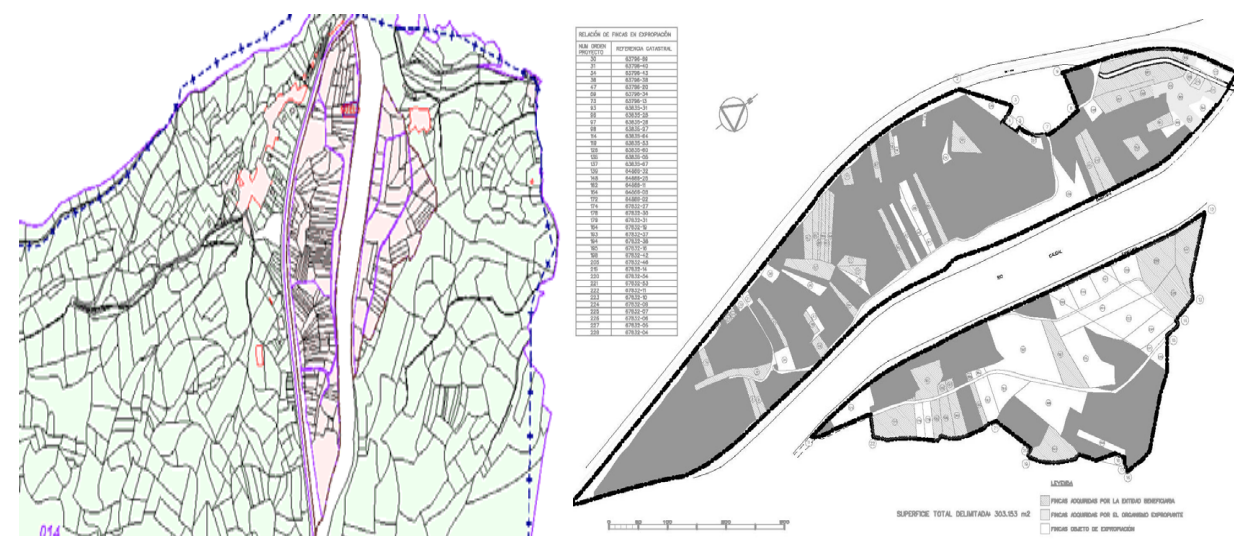

\subsection{Planeamiento}

Respecto al planeamiento, puede ser preciso la modificación puntual de planeamiento general vigente en el municipio, y en todo caso, se redacta un plan parcial, instrumento de desarrollo del plan general, en el que se define la ordenación detallada de una parte del ámbito territorial. Contienen las siguientes determinaciones: Delimitación del área del planeamiento, asignación de usos pormenorizados, fijación de zonas de equipamientos, trazado y características de la red de comunicaciones y su enlace con el sistema general, diseño y trazado de servicios (saneamiento, agua, energía eléctrica...), evaluación económica y plan de etapas de las obras de urbanización.

Para todo ello son imprescindibles una serie de estudios técnicos, como los cartográficos (levantamiento topográfico de la zona de estudio), un plano parcelario (de afección de fincas) y un estudio geotécnico. 
Figura 5: Plano de planeamiento y foto aérea del polígono de Argame (Asturias)

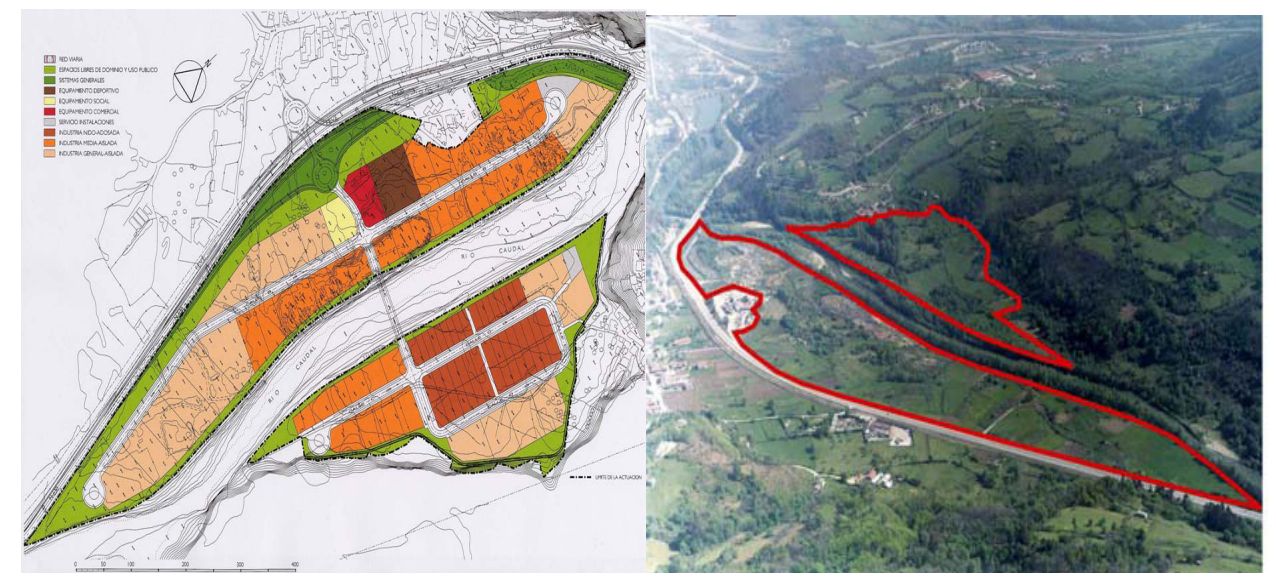

\section{4 Urbanización}

Hay dos fases bien diferenciadas: Redacción de proyecto de urbanización, ajustándose al plan parcial, y la ejecución de las obras, que tendrán gran incidencia en el proceso de transformación del paisaje. Sin embargo, se intenta minimizar sus efectos con medidas correctoras para su restauración ambiental, recogidos en la Declaración de impacto ambiental y en el Estudio de impacto ambiental.

Figura 6: Vista aérea de la ejecución de las obras de urbanización de Argame (Asturias)

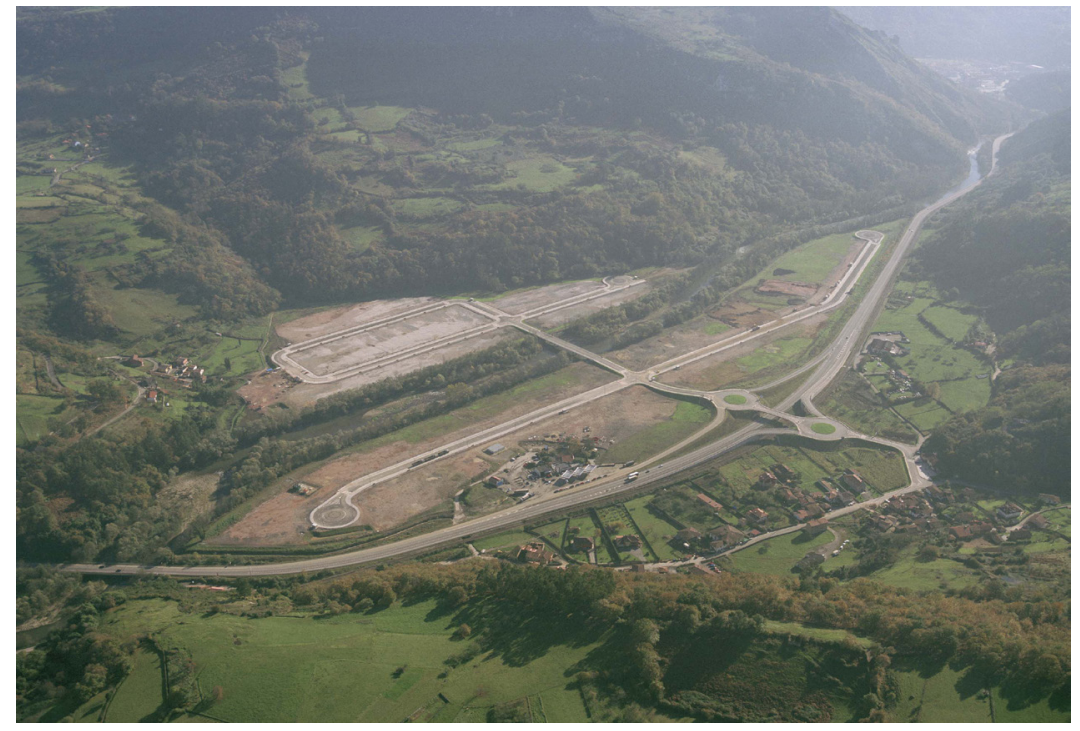


Todas estas medidas que se proponen sirven para la preservación de los recursos y la defensa del medio ambiente, con el fin de salvaguardar los intereses generales desde una perspectiva global e integrada. Asimismo, se deben tener en cuenta los efectos derivados de la actividad proyectada, intentar proteger la salud humana, contribuir mediante un mejor entorno a la calidad de vida, velar por el mantenimiento de la diversidad de especies, conservar la capacidad de reproducción del sistema y evitar, en lo posible, los efectos negativos en los bienes ambientales como el suelo, el agua o en la atmósfera. No debemos olvidarnos de elaborar disposiciones que protejan el paisaje y el patrimonio; de dictar normativa para que se gestionen, de forma adecuada, los residuos, adaptando soluciones tanto en el diseño del proyecto de urbanización como en la ejecución del mismo.

En cuanto a la protección de agua, el proyecto de urbanización se ha de diseñar con un sistema separativo de aguas pluviales y residuales, que permitan recuperar mediante colectores el agua de lluvia y reconducirla a los cauces naturales, o bien utilizarlo para el riego de las zonas verdes; mientras que el colector de residuales se conducirá hacia una estación depuradora, que permita a posteriori su aprovechamiento.

Se tendrá especial atención, en la ejecución de obras con los cursos naturales de agua, como arroyos, río, etc., en los que para evitar vertidos accidentales, se jalonará el cauce delimitándolo correctamente al objeto de evitar derrames y posibles vertidos de sustancias peligrosas (hidrocarburos, aceites) que puedan llegar a afectar la calidad del curso de agua presente en el entorno de actuación. Asimismo, se evitará acumular materiales sobrantes en las proximidades del cauce público. Las obras se realizarán preferentemente en el período estival, cuando el arroyo se encuentra seco, con el fin de paliar posibles afecciones por la emisión de sólidos en suspensión al medio hídrico.

En cuanto a la protección del suelo, susceptible al cambio, ya que es la base para la transformación del paisaje, se tomarán medidas tan simples como la reubicación y acopio de la tierra vegetal mientras que se ejecuta la obra, para la posterior reutilización en la fase de ajardinamiento. Es importante que todos los áridos que intervienen en la fase de obra procedan de canteras legalmente autorizadas, evitando de esta forma riesgos en la seguridad y salud de los trabajadores, como la reducción del impacto para el medio ambiente derivado de una buena gestión y rehabilitación de las zonas mineras.

En lo referente a la protección de la atmósfera, se pueden tomar medidas poco costosas, pero efectivas; como la humectación de los terrenos en condiciones 
desfavorables, evitando que se formen nubes de polvo que afecten a la visibilidad de la circunvalación o a cultivos colindantes; control de emisiones de los escapes de maquinaria. Con carácter previo a su utilización en la ejecución de las obras, se revisarán las emisiones de gases de las máquinas, comprobando que se cumplen los requisitos mínimos exigidos por la Inspección Técnica de Vehículos (ITV) que afecten a emisiones de gases a la atmósfera; control de la velocidad de la maquinaria con señalización adecuada para el control del tráfico durante la ejecución de las obras y recubrimiento, con lonas, en zonas de acopio de materiales.

La idea del paisaje ha estado vinculada a una concepción elitista, en la que la belleza del paisaje se identificaba con espacios especialmente bellos. Esta idea del paisaje ha ido también evolucionando. En este sentido el paisaje es una realidad material e inmaterial, que puede diferir señaladamente según la sensibilidad y valores del observador. El paisaje por otra parte varía de forma constante, por el efecto de la luz o por la constante intervención humana, y es por ello por lo que se deben de tomar ciertas precauciones en el desarrollo y acondicionamiento de la obra. Así, es conveniente la adecuación paisajística de las franjas paralelas a la carretera, creando una "pantalla vegetal" con la disposición de los marcos y densidad de plantación, con gran variedad de especies. De esta manera, transcurrido un tiempo de asentamiento, se constituirá una pantalla natural que mitigará el impacto visual del nuevo polígono en el paisaje. De la misma forma se ajardinarán y restaurarán los márgenes de arroyo con especies autóctonas, que se adapten bien al medio. Con el objetivo de facilitar al máximo la recogida de agua, en el momento de la plantación, se realizarán alcorques y se irán colocando tubos protectores contra roedores en aquellas especies que por su tamaño en el momento de la plantación, sean susceptibles de ataques.

Junto con el paisaje natural se debe de dar también protección al paisaje cultural, al Patrimonio artístico y cultural, que representa la identidad de un lugar y un pueblo. En este sentido, se llevará a cabo un control arqueológico, y en caso de aparición de restos de interés, se comunicará de inmediato al organismo competente, previa paralización de las obras.

Existen otros factores que se tendrán en cuenta, como la protección de los niveles acústicos durante la ejecución de la obra. A tal efecto, se comprobará que todas las máquinas llevan silenciadores y que la velocidad de vehículos y máquinas sea la adecuada. En cuanto a la contaminación lumínica, se atenderá a la legislación vigente, si bien las luminarias instaladas en las vías públicas deberán tener, como máximo un $15 \%$ de emisión de flujo hemisférico superior. El conjunto de la instalación de alumbrado, se adaptará a lo recomendado en el articulado de 
la propuesta de ordenanza municipal de alumbrado exterior para la protección del medio ambiente mediante la mejora de eficiencia energética.

Otro factor sumamente importante para el medio ambiente, es la gestión de residuos. Los residuos generados durante la fase de obra, se trasladarán al destino conveniente en función de la naturaleza de dichos residuos y de acuerdo con la legislación vigente. Los productos y subproductos se gestionarán en función de su catalogación en el catálogo internacional de residuos. Los escombros se trasladarán a vertederos autorizados, y los residuos procedentes del mantenimiento de maquinaria serán retirados por un gestor autorizado de residuos peligrosos. Es conveniente realizar una separación de todos aquellos residuos reciclables que se trasladarán al punto limpio más cercano.

Destacamos la página del Ministerio de Agricultura, Alimentación y Medio Ambiente, donde podemos encontrar la recopilación de información de prevención y gestión de residuos en España (http://www.magrama.gob.es/es/calidad-y-evaluacion-ambiental/temas/prevencion-y-gestion-residuos/).

\section{CONCLUSIONES}

Al concepto de sostenibilidad, entendida como la suma de al menos tres dimensiones que se dan en un territorio; a saber, el social, el económico y el ambiental, no debemos olvidar la dimensión urbanística, y la que resulta de su desarrollo, ya que mediante ella es posible realizar análisis a medio y largo plazo que permita identificar escenarios y tendencias en el crecimiento industrial y/o residencial de una ciudad, inseparables en la estructura urbana.

El proceso a seguir en la transformación de suelo a uso industrial con principios y criterios de sostenibilidad debe de ser objetivo primordial en cualquier actuación urbanística, si pretendemos reconstruir y/o dar continuidad la calidad inicial, dado que los ecosistemas muestran cada vez menor resistencia al impacto de las actividades humanas, y en temas urbanísticos el proceso es el mismo, ya que la ciudad, el territorio, actúa como el agente (ecosistema artificial) que ejerce presión sobre los ecosistemas naturales. El cumplimiento de las medidas expuestas en la fase de urbanización de esta comunicación ayuda, sin duda, a conseguir este objetivo. 


\section{BIBLIOGRAFÍA}

- Brundtland, G.H. (1987). Our common future. Oxford University Press.

- Carrizos A, J. (1993). Colombia, de lo imaginario a lo complejo. Reflexiones y notas acerca de ambiente y desarrollo y paz. Bogotá: Universidad Nacional de Colombia/Instituto de Estudios Ambientales.

- Comisión Europea (1996). "Manual sobre evaluación ambiental de planes de desarrollo regional y programas de los fondos estructurales de UE". Anexo IV: Indicadores. Dirección General XI, Medio Ambiente, seguridad nuclear y protección civil.

- Durán, D.; de Marco, G.; Lara, A.; Sassone, S.; Daguerre, C. (1993). Geografía de la Argentina. Buenos Aires: Ed. Troquel.

- Ekins, P. (1986). The Living Economy. London/ New York: Routledge and Kegan Paul.

- Gómez Orea, D. (1994): Ordenación del territorio: una aproximación desde el medio físico.

- Maza Vázquez, F. (2003). Planeamiento urbanístico en el Corredor del Henares de Guadalajara: Una perspectiva desde el desarrollo sostenible. Universidad de Alcalá (España)

- Pearce, D.W. (1989). Blueprint for a green Economy. London: Earthscan.

- Programa de Naciones Unidas (1996). Indicadores de desarrollo sostenible. Marco y metodología. Nueva York: ONU.

- Solow, R. (1992). "An Almost Practical Step Foward Sustainability". Conferencia en Resorces for the Future, XV Aniversario de RFF, 8/10/92.

- Soriano Sanz, M.L. (2010). "Los sistemas de información geográfica como herramienta de planificación sostenible"Curso Superior de Estudios Territoriales y Urbanísticos. España: INAP/Ministerio de la Presidencia. 